\title{
BREVE PANORAMA DA DIVULGAÇÃO CIENTÍFICA BRASILEIRA NO YOUTUBE E NOS PODCASTS
}

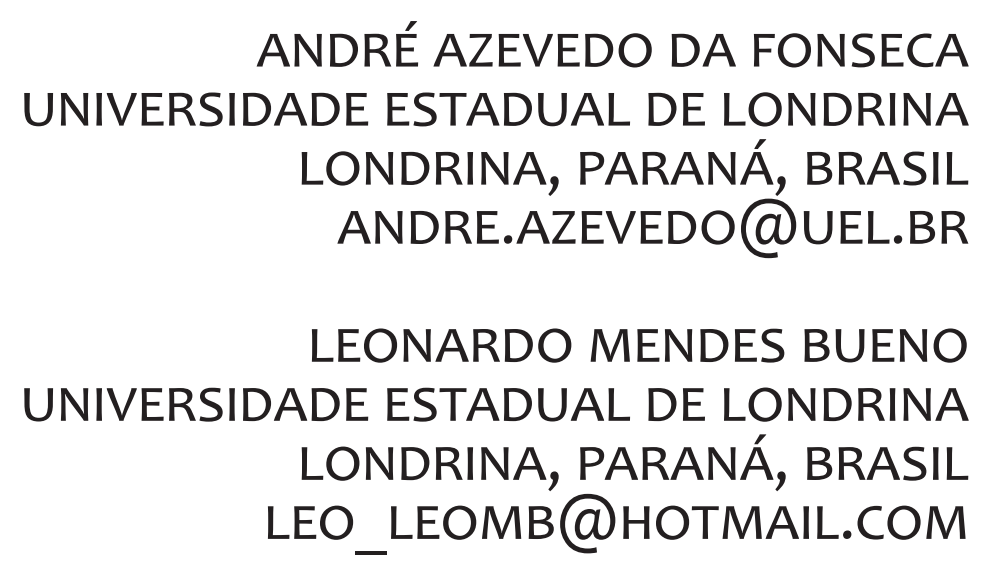




\section{BREVE PANORAMA DA DIVULGAÇÃO CIENTÍFICA BRASILEIRA NO YOUTUBE E NOS PODCASTS}

Resumo: AA partir da análise do contexto histórico da ciência no país, essa pesquisa exploratória quali-quantitativa investiga o panorama de iniciativas de divulgação científica no YouTube e nos podcasts. Os métodos empregados foram a pesquisa bibliográfica e análise documental, com dados sistematizados no software Tableu Public. Foi constatado que os canais com maior número de visualizações e inscritos se dedicam à divulgação científica de temas gerais e de interesse multidisciplinar. Os canais institucionais, por sua vez, são os que mais publicam vídeos. Também foi constatado um movimento de profissionalização dos podcasts, pois a maioria possui site, descrição e está ancorado em uma plataforma de distribuição. Enquanto no YouTube a maioria dos divulgadores são homens do Sudeste, os podcasts têm mais diversidade.

Palavras-chave: Divulgação científica; Podcast, YouTube; Internet.

\section{BREVE DESCRIPCIÓN DE LA COMUNICACIÓN CIENTÍFICA BRASI- LEÑA EN YOUTUBE Y PODCASTS}

Resumen: A partir del análisis del contexto histórico de la ciencia en Brasil, esta investigación exploratoria cuali-cuantitativa indaga en el panorama de las iniciativas de divulgación científica en YouTube y podcasts. Los métodos empleados fueron la investigación bibliográfica y el análisis de documentos, con datos sistematizados en el software Tableu Public. Se encontró que los canales con mayor número de visualizaciones y suscriptores se dedican a la divulgación científica de temas generales y de interés multidisciplinario. Los canales institucionales, a su vez, son los que más publican videos. También hubo un movimiento para profesionalizar los podcasts, ya que la mayoría tienen sitio web, descripción y están anclados en una plataforma de distribución. Mientras que en YouTube la mayoría de los promotores son hombres del sureste, los podcasts tienen más diversidad. Palabras clave: Difusión científica; Podcast, YouTube; Internet.

\section{BRIEF OVERVIEW OF BRAZILIAN SCIENCE COMMUNICATION ON YOUTUBE AND PODCASTS}

Abstract: Based on the analysis of the historical context of science in Brazil, this exploratory quali-quantitative research investigates the panorama of scientific dissemination initiatives on YouTube and podcasts. The methods employed were bibliographic research and document analysis, with data systematized in the Tableu Public software. It was found that the channels with the highest number of views and subscribers are dedicated to the scientific dissemination of general topics and of multidisciplinary interest. Institutio- 
nal channels, in turn, are the ones that most publish videos. There was also a movement to professionalize podcasts, as most have a website, description and are anchored in a distribution platform. While on YouTube the majority of promoters are men from the Southeast, podcasts have more diversity. Keywords: Scientific dissemination; Podcast, YouTube; Internet.

\section{INTRODUÇÃO}

Como pensar os caminhos da ciência e da divulgação científica em um país repleto de desigualdades sociais e onde os cortes públicos nas áreas de ciências e educação têm sido cada vez mais frequentes? Massarani e Moreira (2002) nos indicam alguns caminhos a partir da investigação de aspectos históricos da ciência no Brasil desde o período colonial.

A princípio, as necessidades do governo português relacionadas à ciência e tecnologia estavam conectadas aos interesses políticos, econômicos e militares. Apenas no início do século XIX houve uma manifestação consistente de comunicação científica no Brasil. Nesse período, o país ganhou os primeiros periódicos em que notícias relacionadas à ciência foram escritas: A Gazeta do Rio de Janeiro e O Patriota.

Segundo Massarani e Moreira (2002), em uma análise do catálogo da Biblioteca Nacional, foi constatado que 300 dos 7000 periódicos criados no Brasil no século XIX eram de alguma forma relacionados à ciência. Além disso, houve um crescimento acentuado na criação desses veículos a partir de 1860. O editorial da edição de 1876 da Revista do Rio de Janeiro, por exemplo, já defendia que uma das formas mais eficazes para estimular a educação e o desenvolvimento social era popularizar as ciências, as letras, as artes, a agricultura, o comércio e a indústria (MASSARANI e MOREIRA, 2002).

Já no século $X X$, foram criadas instituições que contribuíram significativamente para a difusão científica no país. Este período foi rico em termos de diversificação de iniciativas de comunicação de ciência no Brasil. Revistas populares foram criadas para atendar a demanda. Mais tarde, canais da televisão aberta, como na Rede Globo e na TV Cultura, desenvolveram diversos programas de caráter científico e educacional.

Entre o final do século XX e início do século XXI, a criação de fundações de amparo à pesquisa e a proliferação de eventos que fomentavam o interesse pela ciência, tal como olimpíadas de diferentes áreas, tem contribuído nesse contexto. Além disso, museus e centros de interação com o público, TVs universitárias educativas, espaços exclusivos para a ciência em jornais, 
bem como jornalistas especializados atuando nos maiores periódicos do país e, mais recentemente, a criação de redes de cientistas e comunicadores de ciência na internet também são fatores importantes no estímulo à cultura científica.

Ainda assim, boa parte dos brasileiros não conhece seus cientistas. Segundo Massarani e Moreira (2016), os desafios para a comunicação científica podem ser divididos em três dimensões: ampliar o alcance social; criar políticas públicas que favoreçam as universidades e apoiem a produção científica nacional; melhorar a qualidade da comunicação científica que está sendo produzida.

Um dos pesquisadores pioneiros que desenvolveu teorias e práticas da cultura científica no Brasil, Carlos Vogt (2003), ressaltou a relação dinâmica entre ciência e cultura.

A ideia de que o processo que envolve o desenvolvimento científico é um processo cultural, quer seja ele considerado do ponto de vista de sua produção, de sua difusão entre pares ou na dinâmica social do ensino e da educação, ou ainda do ponto de vista de sua divulgação na sociedade, como um todo, para o estabelecimento das relações críticas necessárias entre o cidadão e os valores culturais, de seu tempo e de sua história (VOGT, 2003).

No contexto das transformações aceleradas na comunicação a partir do desenvolvimento da Internet e das redes sociais, o objetivo da presente pesquisa é identificar o panorama das iniciativas de divulgação científica no YouTube e nos podcasts brasileiros. Para isso, a partir dos dados fornecidos por uma lista pública' de iniciativas de divulgação científica nas redes sociais, estabelecemos um conjunto de categorias para compreender os temas, as áreas do conhecimento e o alcance dessas iniciativas.

Essa pesquisa de caráter quali-quantitativo se vale, inicialmente, do método de pesquisa bibliográfica. Os critérios foram estabelecidos a partir de alguns parâmetros. No parâmetro temático, selecionamos artigos que analisaram canais de ciência no YouTube e podcasts, delimitando as buscas ao contexto brasileiro. No parâmetro de fontes, consideramos artigos publicados em periódicos, dissertações e teses, bem como textos analíticos em

1 Disponível em: https:/github.com/DivulgacaoCientifica/divulgacaocientifica/blob/ master/YOUTUBE.md. Acesso em: 12 de set. 2020. 
revistas de divulgação científica, de forma a priorizar trabalhos produzidos nos últimos cinco anos. Assim, "a pesquisa bibliográfica possibilita um amplo alcance de informações [...] auxiliando também na construção, ou na melhor definição do quadro conceitual que envolve o objeto de estudo proposto" (GIL apud LIMA, 2007).

Em seguida, a partir de um mapeamento de iniciativas de divulgação científica no YouTube e em podcasts realizado de forma colaborativa, definimos categorias para sistematizar os dados. Em relação aos canais do YouTube, consideramos o número de inscritos no canal, número de visualizações, número de vídeos publicados e os respectivos temas. Em relação aos podcasts, consideramos o número de faixas produzidas, se possui descrição, se está ancorado em alguma plataforma ou em um site próprio e, por fim, seus respectivos temas.

A pesquisa se concentrou em podcasts e vídeos no Youtube porque estas tecnologias se firmaram como as principais alternativas contemporâneas em áudio e vídeo para a produção de conteúdos aprofundados de divulgação científica. Neste trabalho, portanto, não foram abordados stories no Instagram, vídeos curtos do TikTok ou as iniciativas de divulgação científica no Twitter. Para nos concentrarmos na produção em áudio e vídeo, também não foram abordados os tradicionais blogs de ciência. Assim, espera-se contribuir no entendimento da atual cena de divulgadores científicos naquelas plataformas e contribuir para futuras pesquisas.

\section{DIVULGAÇÃO CIENTÍFICA NO YOUTUBE}

O YouTube tem oferecido uma oportunidade interessante ao movimento de divulgação científica na Internet. A plataforma é o maior site de compartilhamento de vídeos do mundo, comportando conteúdos de diversos assuntos e temáticas. O Brasil é um dos principais consumidores dessa plataforma. Graças a isso, a divulgação científica encontrou um espaço de grande reverberação, com relativa demanda de público. Naturalmente, como apontado por Silva et al (2019), há muitas contradições no modelo de negócio das plataformas, fazendo com que divulgadores tenham que enfrentar uma série de problemas, tais como ataques ideológicos e baixa remuneração de seu trabalho. Além disso, é preciso considerar o encantamento provocado pelas propagandas de tecnologia, que costumam prometer mais benefícios do que realmente entregam (FONSECA, 2019a). Contudo, ao lado dos desafios, há muitas oportunidades. 
Para se consolidar como youtuber, o divulgador fomenta comunidades com seu público e com outros canais, que podem estar atreladas a outras redes sociais como Instagram, Twitter ou Facebook, por exemplo. Essas comunidades se relacionam com o canal de forma ativa, compartilhando e distribuindo seu conteúdo. Essencialmente, um divulgador científico no YouTube deve tornar a ciência atrativa para seu público, estimulando não apenas visualizações, mas engajamento e disseminação orgânica. A grande virtude envolvendo o YouTube é a facilidade de inscrição, produção e distribuição de conteúdo. Se considerarmos que, de acordo com uma pesquisa de mercado do Google, youtubers já são mais influentes do que jornalistas (FONSECA, 2019C), fica evidente o poder da plataforma.

Reale e Martyniuk (2016) sustentam que a divulgação científica através do YouTube é uma excelente ferramenta para democratizar o conhecimento científico. Vídeos de ciência proporcionam a muitos jovens o primeiro contato com assuntos importantes para a formação cidadã. Esse movimento de aproximação em que o professor sai do seu "pódio doutoral" e se coloca mais próximo do espectador se dá quando ele faz uma conexão entre os gostos de ambos.

A construção de sentido ligada a elementos sensíveis, pensada no imaginário do destinatário, propõe um caminho de formação do gosto pela ciência. $O$ apelo ao sensível é fundamental para a construção de uma comunicação normativa em diálogo com o outro em busca de um ideal comum: gostar sobre ciência (REALE; MARTYNIUK, 2016).

Além disso, pesquisas como a de Aranha et tal (2017) confirmam que o YouTube pode ser também um aliado no ensino de ciências. Ele observou as maneiras pelas quais os vídeos de divulgação científica no YouTube podem ser utilizados no ensino e constatou que "canais aliados a outras metodologias ou estratégias educacionais, planejadas frente às competências almejadas, podem auxiliar os professores em suas novas tarefas" (ARANHA et al, 2017, p. 14).

\section{SCIENCE VLOGS BRASIL}

Uma das comunidades mais importantes criadas por divulgadores científicos brasileiros foi o Science Vlogs Brasil (SVBR). O SVBR atua como um selo de qualidade para os youtubers que abordam temas de ciência ou as- 
suntos relacionados. Para um canal obter a certificação, ele deve, em primeiro lugar, ser indicado por um parceiro SVBR. Em seguida, o conselho SVBR avalia os indicados, convidando especialistas para avaliar o canal. Por fim, os conteúdos são avaliados pela comunidade de canais da rede.

Velho (2019) analisou canais do SVBR e concluiu que os objetivos propostos pelo projeto, tal como "maximizar o alcance dos vídeos de cada youtuber na plataforma através da citação cruzada (feita pelos colegas de projeto), alcançar maior visibilidade do grupo como um todo, [...] e garantir confiabilidade da informação veiculada" (SCIENCEBLOGS apud VELHO, $2016,120)$ são desafios a serem ainda alcançados. Considerando a intensa disputa por atenção no contexto da plataforma, é inegável que canais de entretenimento e notícias têm mais alcance. Além disso, apesar de estimular a colaboração, o YouTube monetiza a visibilidade de canais em um sentido individual, e não coletivo. O último ponto se relaciona à dificuldade estrutural de alcance de vídeos com informações científicas e confiáveis, visto que o algoritmo da plataforma gera maior visibilidade para conteúdos sensacionalistas que promovem amplo engajamento (FONSECA, 2019b).

Ramos (2017) constatou que inúmeros canais no YouTube produzidos por criadores sem formação científica se dedicam a debater temas que, por princípio, exigem subsídio acadêmico. Para verificar a confiabilidade de informações de teor científico veiculadas na internet, portanto, ela analisou cinco canais certificados pelo SVBR e cinco canais não certificados. Os critérios de qualidade foram estabelecidos a partir dos moldes descritos por Tomaél (2001). Nesse sentido, chegou-se à conclusão de que a maioria dos vídeos analisados não possui orientação ou padronização em relação a possibilidade de falseamento de informações. Entretanto, Ramos (2017) constatou que há um movimento no sentido de garantir a qualidade dos canais certificados pelo SVBR, incluindo no que diz respeito ao vídeo, som e pós-produção.

Para identificar questões relacionadas à profissionalização, Reale (2018), analisou 197 canais de divulgação científica no YouTube e concluiu que mais da metade $(58,98 \%)$ não possuía vídeo introdutório; 91,9\% possuíam descrição de seus objetivos; $66 \%$ estavam vinculados a um selo de qualidade; $53,81 \%$ trabalhavam em grupo; 52,3\% possuíam formação acadêmica; e 68,53\% atuavam com uma produção de conteúdo “frio" - ou seja, aquele que discute temas mais amplos, sem vínculo imediato com temas do momento

Na mesma linha, Velho (2019) também traçou um perfil dos youtubers 
divulgadores de ciência; entretanto, se restringiu a uma parcela dos participantes do SVBR. Ela concluiu que são majoritariamente graduandos ou pós-graduandos, homens, entre 18 e 35 anos, residentes no Sudeste e que tratam de assuntos relacionados às Ciências Exatas e Biológicas.

Utilizando dados de Bärtl (2018), Borghol et al (2012), Allgaier (2016) e Welbourne \& Grant (2015), Velho (2019) observa que, dos vídeos publicados no YouTube, os mais antigos são mais populares em relação aos mais novos; os identificados com um maior número de palavras-chave são, em geral, mais acessados; os que veiculam conteúdos polêmicos tendem a exibir maior engajamento e número de visualizações; e os apresentados em ritmo mais rápido são mais visualizados. Ainda, a partir de Morcillo, Czurda e Trotha (2016), Velho (2019) observa que o poder narrativo dos apresentadores, somado ao uso competente das técnicas de edição de vídeo, são essenciais para a popularidade de vídeos de divulgação científica.

\section{PODCAST E DIVULGAÇÃo CIENTÍFICA}

Podcasts são produtos de comunicação em áudio distribuídos em plataformas na Internet e consumidos sob demanda, diferentemente do modelo de transmissão dos programas de rádio tradicionais. As vantagens na produção e consumo de podcasts vão desde o baixo uso de dados nas etapas de transmissão até a facilidade de consumo de conteúdos enquanto o ouvinte realiza outras tarefas. Além disso, o podcast é uma alternativa que pode ser acessada a qualquer momento nos mais diversos dispositivos tecnológicos, tal como smartphones, computadores ou tablets. O nome tem origem nas palavras iPod, que é o dispositivo de arquivo MP3 da empresa Apple, e Broadcast, que significa "transmissão" ou "radiodifusão".

Para a produção de um podcast, o criador do conteúdo precisa de poucos recursos técnicos: um microfone simples e um programa de edição de áudio. Apesar de o formato ter sido inaugurado por produtores independentes, atualmente tem sido amplamente explorado por veículos de comunicação da mídia tradicional. Podcasts podem ser veiculados em um site próprio ou em plataformas específicas de streaming, que são muito acessadas.

Segundo Luiz e Assis (2010), a interatividade faz com que o conceito tradicional de receptor passivo das mídias de massa seja mais uma vez ultrapassado. Assim, a divulgação científica atinge novos parâmetros quando aliada ao podcast. E assim, com auxílio da própria comunidade, pode contribuir ainda mais para o combate às fake news e a pseudociência disseminada 
na internet (MORAIS, et tal, 2019).

Gums et al (2019) mapearam 63 podcasts de divulgação científica através de uma pesquisa exploratória de caráter quantitativo. Eles utilizaram o mapeamento anterior feito pela pesquisadora e colaboradora do podcast de divulgação científica Dragões de Garagem, Gabriela Barbosa Sobral de Oliveira, e se basearam no período de atividade dos podcasts entre janeiro e abril de 2019 . Foi constatado que a maioria dos podcasts $(53,33 \%)$ são apresentados por uma equipe constituída de homens e mulheres. Quando se trata de um apresentador único, homens respondem por cerca de 38,33\% e mulheres 8,33\%. Grande parte dos podcasts são independentes (70\%). Mas $13 \%$ são vinculados a universidades. Também se constatou que $51 \%$ dos produtores de podcast são pesquisadores, $16 \%$ são profissionais da área em que atuam, $8 \%$ são mistos entre profissionais e pesquisadores, $3 \%$ são jornalistas e $3 \%$ são feitos pelo público em geral. Naturalmente, podcasts são produções muito dinâmicas. Por isso, esses dados representam um momento específico do desenvolvimento dessa comunidade.

Em relação às áreas do conhecimento em que esses podcasts estão situados, Gums et al (2019) concluíram que a maioria (32\%) têm conteúdo multitemático. Em relação à periodicidade, constatou-se que apenas $2 \%$ dos podcasts produzem conteúdo inédito diariamente. Na maioria deles não há um cronograma definido. Assim, os autores concluem que o mais marcante nos podcasts é o desafio da reformulação do discurso científico, historicamente presente na questão da divulgação científica. Ainda, nos podcasts analisados destacam-se os cientistas que, "antes tidos como fontes de informação dos jornalistas - passaram a atuar diretamente na produção do conteúdo" (GUMS et al, 2019, 13).

Para Mendes (2019), o podcast tem um grande potencial de divulgação científica graças ao seu crescimento, facilidade de produção, distribuição e consumo, além da linguagem acessível. O podcast pode ser ouvido individualmente ou em grupo sem muitos desafios tecnológicos, além de ser uma ótima ferramenta para ser utilizada em sala de aula por um professor.

\section{PROCEDIMENTOS METOdOLÓGICOS}

Para coletar os metadados dos canais do YouTube da lista pública e colaborativa disponível na plataforma GitHub, foi utilizado o software "YouTube Data tools" (RIEDER/sd). O pograma disponibiliza uma ferramenta de análise precisa das informações pertinentes à esta pesquisa. Os critérios utilizados 
para análise foram o número de visualizações do canal, número de inscritos, número de vídeos publicados e eixo temático. Inicialmente analisamos 249 canais do YouTube, com alguns deles participando do SVBR; entretanto, sete canais ou foram excluídos ou estavam repetidos na lista. Dessa forma, chegamos ao número de 242 canais. Desses, apenas os que tinham a palavra "channel" em sua URL foram analisados pelo software; assim, todos os outros canais que possuíam a palavra "user" em sua URL, foram analisados manualmente.

Já em relação à coleta de dados dos podcasts, inicialmente a lista continha 96 títulos; entretanto, oito deles não puderam ser analisados porque as URLs estavam inativas. Desse modo, coletamos informações de 88 podcasts e analisamos: o número de faixas produzidas, se possui descrição, se está ancorado em alguma plataforma e se possui site próprio. Os dados foram coletados e tabulados em gráficos utilizando o software Tableau Public, que possui diversas ferramentas para comparação e cruzamento dos dados. A coleta nos canais de YouTube e nos podcasts foi efetuada no período de 23 de maio até 29 de junho de 2020. Portanto, representa um retrato deste período.

É importante salientar que o biólogo e divulgador científico Átila lamarino conquistou grande relevância durante a pandemia do novo coronavírus. Além de participar do canal Nerdologia, lamarino passou a publicar vídeos regularmente no seu próprio canal a partir de agosto de 2019. E depois de uma live no dia 20 de março de 2020, quando discutiu as projeções da pandemia, o canal cresceu exponencialmente, alcançando, até novembro de 2020, a marca de 1,3 milhão de inscritos. Por isso, no presente artigo, realizamos uma nova coleta de dados entre os dias 2 e 6 de novembro para registrar o crescimento deste canal, que não estava contemplado na primeira versão da pesquisa.

\section{PROCEDIMENTOS METODOLÓGICOS}

\subsection{Dados do Youtube}

Entre os dados encontrados observamos que a maioria dos canais de divulgação científica do YouTube $(35,39 \%)$ apresenta conteúdos gerais, podendo assim transitar entre diversos temas. Exemplos de canais com essa dinâmica são o Nerdologia, Nostalgia e Manual do Mundo, além de canais institucionais como o Canal USP, LabI UFScar, TV UFG, dentre outros. Para organizar os canais em temas, à priori seguimos a classificação contida na 
própria lista do GitHub. Depois utilizamos a divisão de áreas da Coordenação de Aperfeiçoamento de Pessoal de Nível Superior (CAPES).

Gráfico 1: Porcentagem de temas por canal.

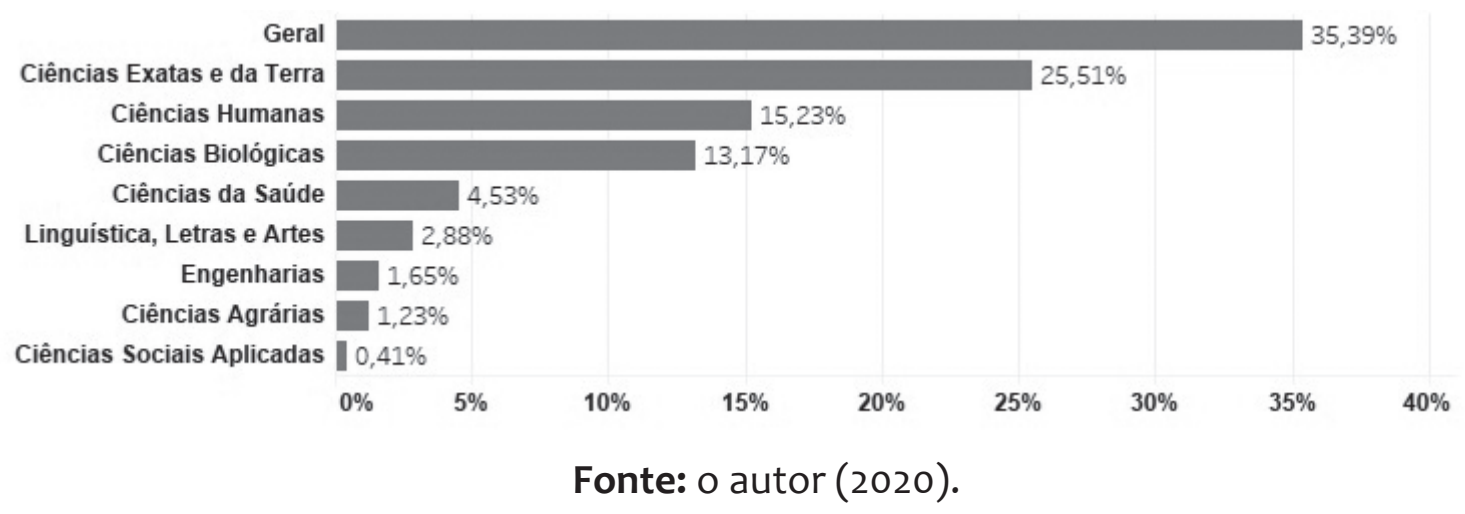

Dessa forma, o canal que antes estava classificado como conteúdo de "História", passou a ser classificado como "Ciências Humanas", por exemplo. Deste modo, podemos constatar que um quarto $(25,51 \%)$ dos canais produz conteúdo sobre Ciências Exatas e da Terra, apresentando o segundo maior tema a ser abordado. Também é possível observar que menos de $1 \%$ dos canais tem como foco o conteúdo voltado às Ciências Sociais Aplicadas.

Além disso, podemos observar que o canal Manual do Mundo, juntamente com o canal Nostalgia, são os maiores do Brasil em número de inscritos. Seguido do canal Nerdologia, que também faz divulgação científica com conteúdos diversos. Outro dado importante é que dois canais de Saúde estão entre os com mais inscritos: Drauzio Varella e Tua saúde - o que indica a grande busca por informações médicas na Internet.

Gráfico 2: Canais com mais escritos.

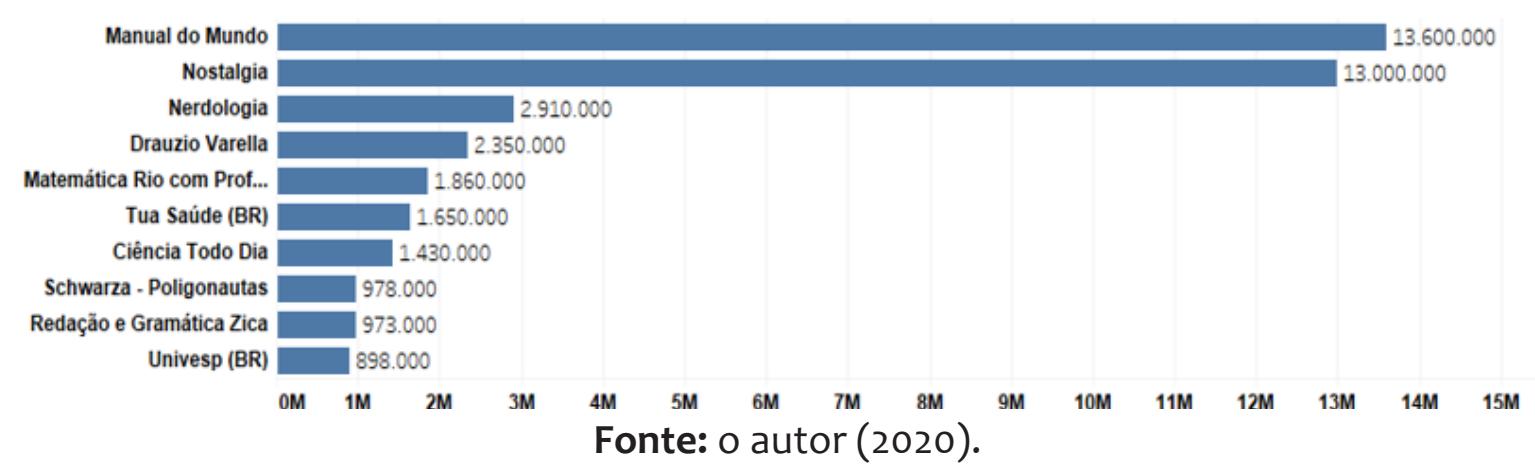

Sobre os canais mais acessados no YouTube, podemos observar que o Manual do Mundo possui mais de dois bilhões de visualizações, seguido pelo canal Nostalgia, com um pouco mais de um bilhão de visualizações. Além 
disso, pode-se observar novamente que os três canais com mais visualizações são aqueles que produzem conteúdo voltado para a divulgação científica com conteúdos gerais. Mais uma vez, vemos os canais sobre saúde, Drauzio Varella e Tua Saúde; entretanto, a lista também contém o canal do Ministério da Saúde, com mais visualizações do que os dois canais citados anteriormente. $O$ canal Univesp aparece como o único de uma instituição de ensino superior a ter mais de 100 milhões de visualizações.

Gráfico 3: Canais com mais visualizações.

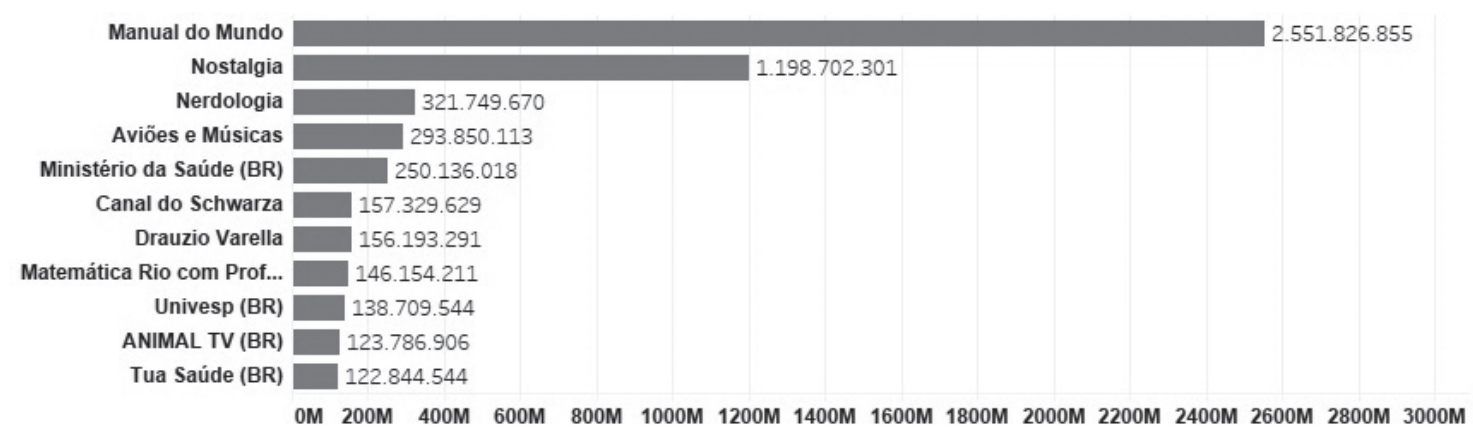

Fonte: o autor (2020).

Em relação aos canais com mais vídeos publicados, podemos observar que a maioria são canais institucionais como TV UFG, Univesp, Canal USP, Ministério da Saúde, TV Escola e LabI UFSCar, respectivamente. Além disso, esses canais também estão dentro dos 35,39\% que fazem parte da categoria Geral.

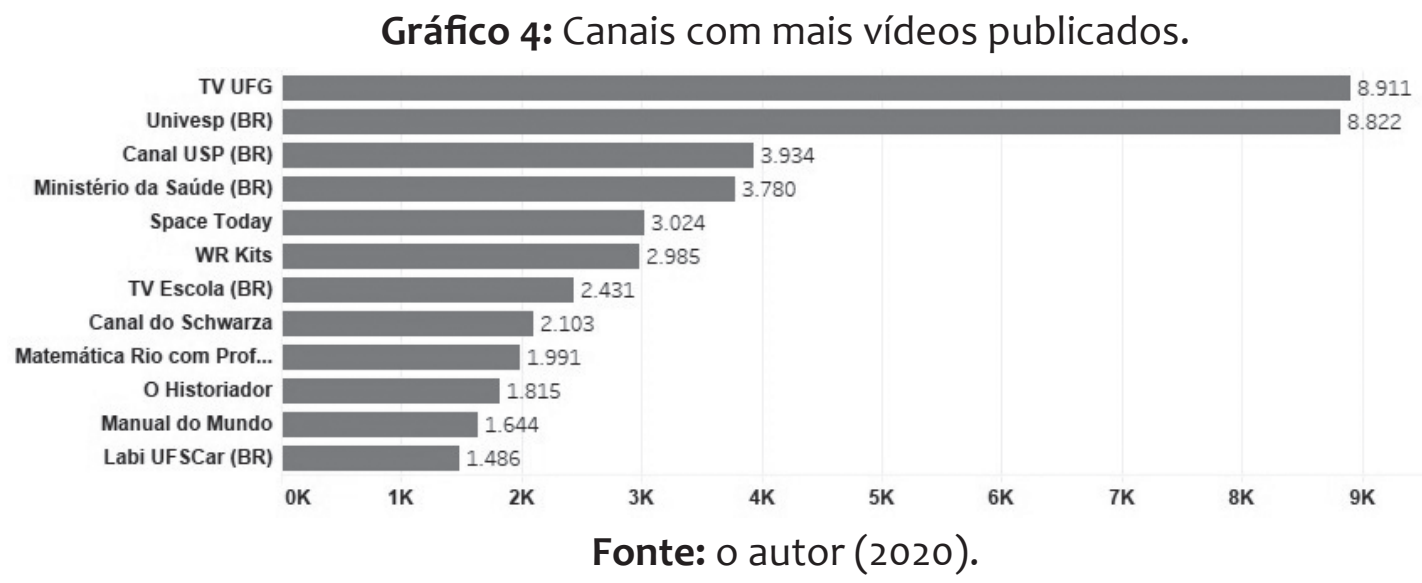

Por fim, nesse gráfico comparativo, constatamos que os canais de tema geral são os que possuem os maiores números, não importa em qual categoria. Além disso, percebe-se que, por mais que as Ciências Sociais Aplicadas tenham o menor número de canais produzindo conteúdo, como visto 
no Gráfico 1, seus números de inscritos e visualizações são maiores que os das Ciências Agrárias, que possui mais vídeos publicados.

Gráfico 5: Número de inscritos, número de visualizações e número de vídeos publicados, divididos por área do conhecimento.

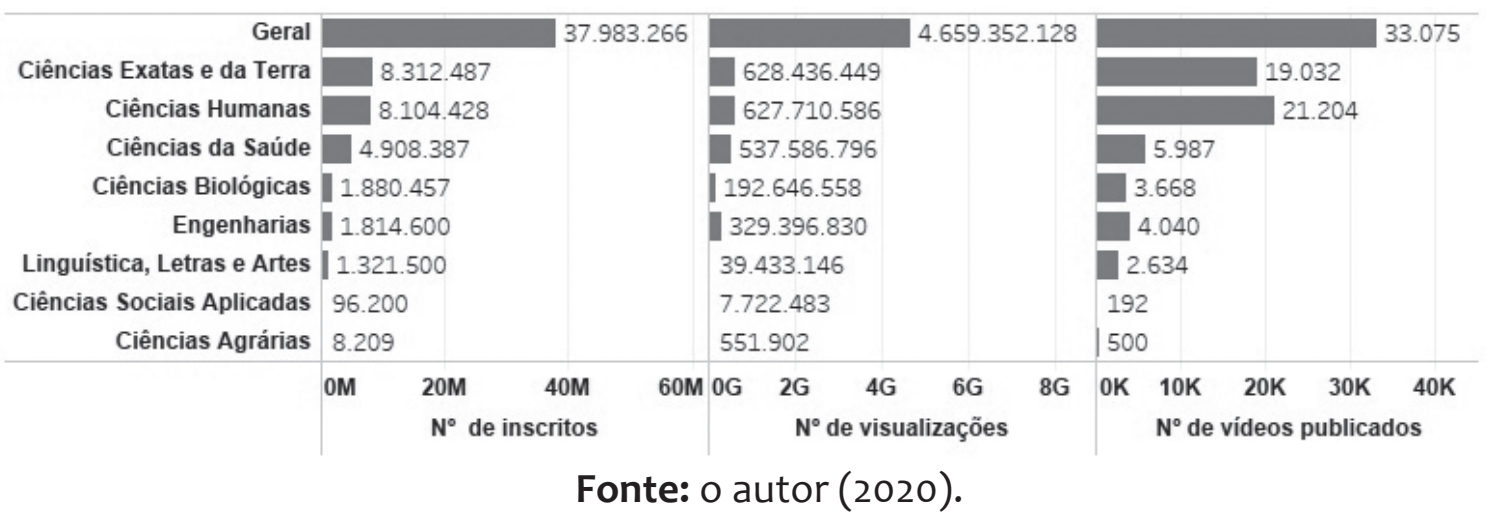

\subsection{Dados dos podcasts}

Sobre os dados dos podcasts de divulgação científica, constatamos que um pouco mais de um quinto deles (20,45\%) não está ancorado em alguma plataforma como Spotify, SoundCloud, Apple podcast, Deezer, Google podcast, dentre outros. Assim, essa parcela de podcasts geralmente só é acessível por um site.

Gráfico 6: Porcentagem de podcasts que estão ancorados em uma plataforma.

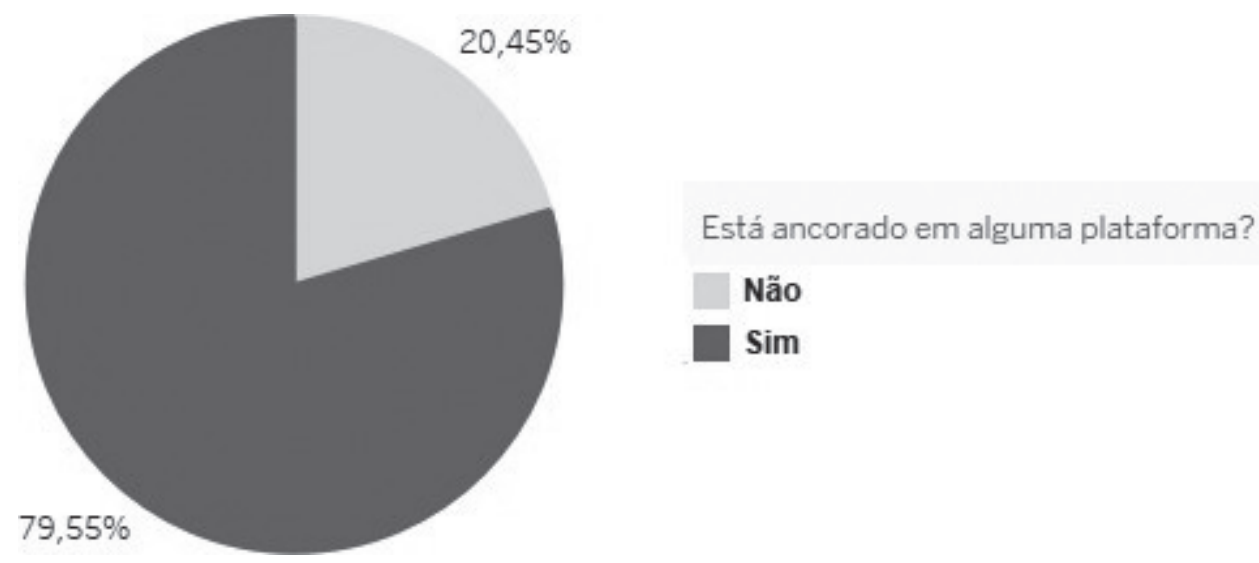

Fonte: o autor (2020).

A maioria dos podcasts (92,05\%) possui um site próprio, tanto aqueles que não ancoram seu produto nas plataformas citadas anteriormente quanto aqueles que fazem essa distribuição. Também constatamos que a maioria dos podcasts analisados contém descrição em algum momento: seja uma aba "Sobre" no site próprio, seja na descrição do Spotify ou outro agrega- 
dor.

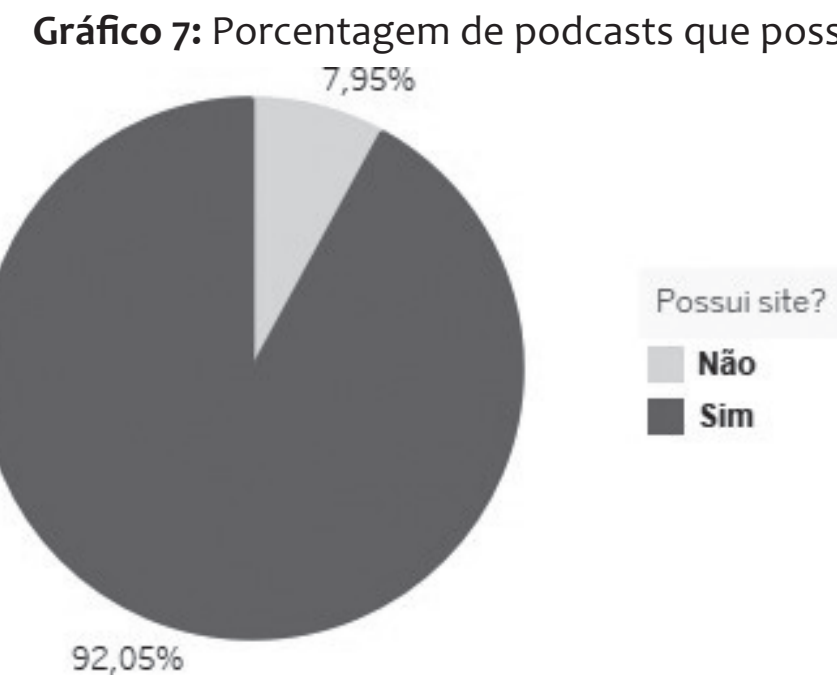

Fonte: o autor (2020).

Algumas descrições continham dados da equipe juntamente com a descrição; outros optavam por ter uma aba específica para isso. A descrição é essencial para que o ouvinte saiba, em linhas gerais, do que se trata aquele podcast.

Gráfico 8: Porcentagem de podcasts que possuem descrição.

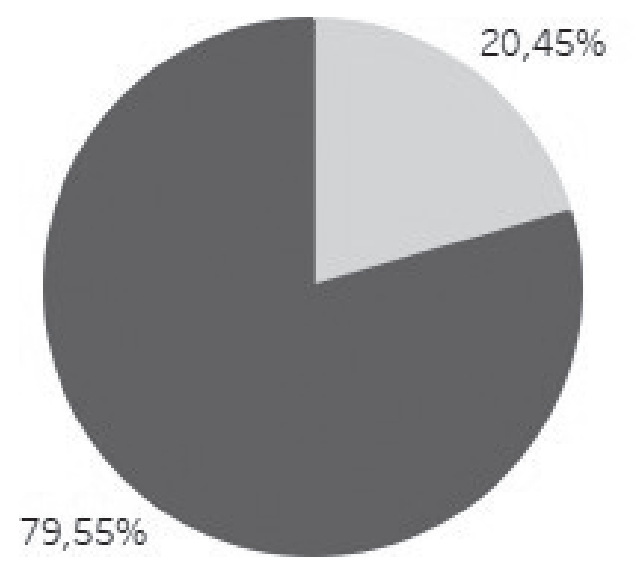

\section{Possui descrição?}

Não

Fonte: o autor (2020).

Por fim, observamos os 15 podcasts com mais faixas produzidas no Gráfico 9. Existem dois podcasts institucionais em evidência: Pesquisa FAPESP e Ciência USP, mostrando o empenho das instituições em comunicar a ciência produzida em seus programas de pós-graduação. Também identificamos que os dois podcasts com mais faixas produzidas têm produção diária. $O$ primeiro trata de tecnologia e o segundo de divulgação científica geral. 
Gráfico 9: Podcastas com mais faixas produzidas.

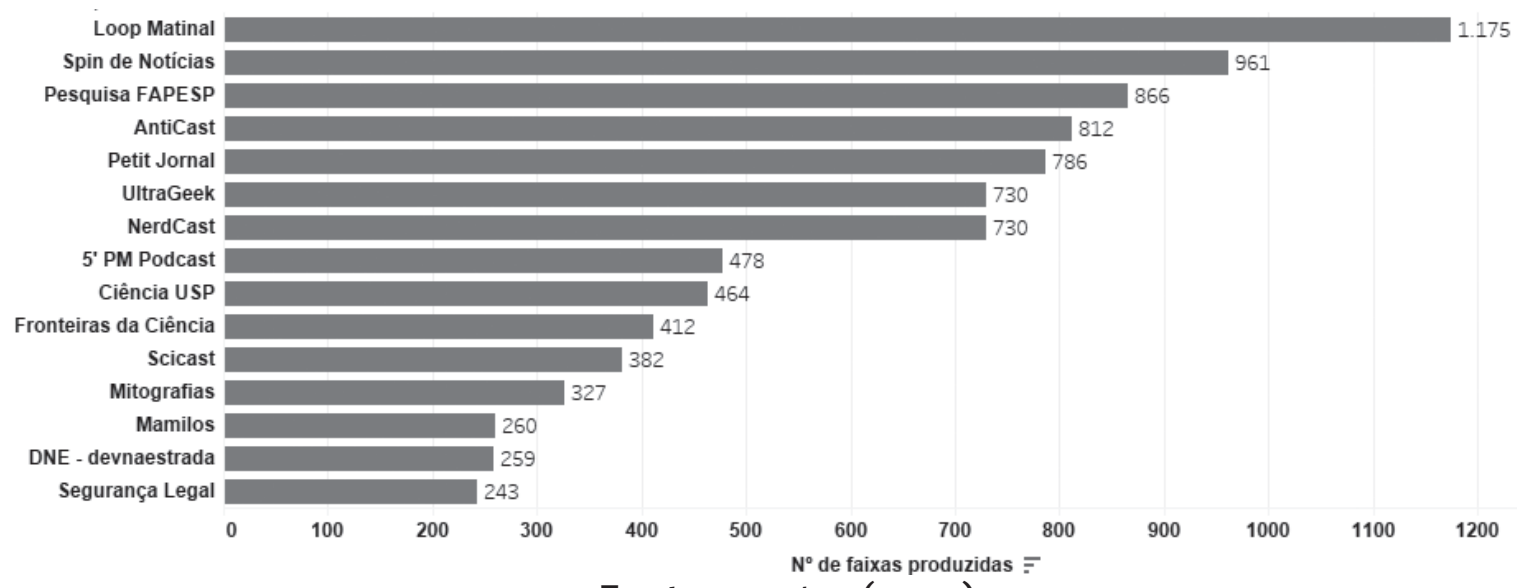

Fonte: o autor (2020).

Esses dados indicam a vitalidade, a diversidade e as características do movimento de divulgação científica nessas plataformas.

\section{CONSIDERAÇÕES FINAIS}

O YouTube oferece aos divulgadores a oportunidade de interagir com sua audiência, fomentando o interesse pela ciência por meio do estímulo à cultura participativa. Além disso, a facilidade de criação de um canal - cujos vídeos não precisam necessariamente de uma produção sofisticada - é uma das características que contribuem para que o YouTube seja uma alternativa viável de divulgar ciência. Existem, ainda, projetos que visam utilizar os vídeos de divulgação científica no YouTube como forma de auxiliar em ambientes pedagógicos, proporcionando uma abordagem criativa e acessível aos estudantes.

Contudo, ainda há muito a avançar. Observamos que, com algumas exceções, a rede Science Vlogs Brasil, por exemplo, ainda é composta majoritariamente por homens, entre 18 e 35 anos, que estão situados no Sudeste. Ou seja, mesmo em iniciativas promissoras existem barreiras geográficas, de gênero e etnia, que são estruturais na sociedade brasileira, e que, se fossem superadas, causariam impactos importantes. Além disso, observamos que a produção de divulgação científica no YouTube se concentra em temas gerais e na área de Ciências Exatas e da Terra. As áreas de Ciências Sociais Aplicadas e Ciências Agrárias são as que menos possuem conteúdos na plataforma. Sobre os canais, constatamos que o Manual do Mundo e o Nostalgia são os que mais possuem inscritos e visualizações, concentrando boa parte da audiência no YouTube. Ainda, observamos que os canais institucionais são 
os que mais possuem vídeos publicados, disponibilizando conteúdos e contribuindo para a comunicação pública da ciência.

No caso dos podcasts, foi observado que a distribuição entre gêneros é mais equilibrada. Em geral, pesquisadores falam sobre conteúdos multitemáticos. O podcast é ainda mais fácil de ser produzido do que conteúdos audiovisuais, pois necessita de poucos recursos técnicos. Entretanto, apenas $2 \%$ deles lançam episódios diariamente. Constatamos que a maioria dos podcasts estão ancorados em plataformas agregadoras como o Spotify e SoundCloud, o que permite maior alcance. Também foi constatado que boa parte dos podcasts possui um site próprio com informações da equipe e com a descrição geral do produto, fazendo com que o ouvinte tenha uma noção prévia dos assuntos. Por fim, observamos que os dois podcasts com mais episódios têm produção diária e tratam de tecnologia e divulgação científica geral, respectivamente. Além disso, observamos que dois podcasts institucionais estão entre os que mais produzem; entretanto, se compararmos ao YouTube, o número de canais institucionais em evidência é maior.

Canais no YouTube e podcasts de ciência têm se consolidado como alternativas viáveis para a comunicação de ciência de forma aprofundada. Esperamos que o mapeamento realizado neste trabalho auxilie novas pesquisas sobre divulgação científica na Internet e contribua com produtores de conteúdo interessados em compreender as métricas relacionadas às suas atuações.

\section{REFERÊNCIAS}

ARANHA, Carolina Pereira et al. O YouTube como Ferramenta Educativa para o ensino de ciências. Olhares \& Trilhas, v. 21, n. 1, p. 10-25, 2019.

BOTTENTUIT JUNIOR, J. B.; Coutinho, C. P. Podcast em Educação: um contributo para o estado da arte. In. CONGRESSO INTERNACIONAL GALEGO PORTUGUÊS DE PSICOPEDAGOGIA, 9. 2007, Coruña. Actas... Coruña: 2007b, p.837-846. Disponível em: https://repositorium.sdum.uminho.pt/bitstream/1822/7094/1/pod.pdf. Acesso em: 04 set. 2020.

CARVALHO Mariela Costa. Divulgação científica no Youtube: narrativa e cultura participativa nos canais Nerdologia e Peixe Babel. In: CONGRESSO BRASILEIRO DE CIÊNCIAS DA COMUNICAÇÃO, 39. 2016, São Paulo. Anais... São Paulo: Intercom, 2016. p.1-12. Disponível em: https://portalintercom.org.br/anais/nacional2016/resumos/R11-2014-1.pdf. Acesso em: 4 set. 2020.

FONSECA, André Azevedo da. Do horror tecnocrático ao encanto da máquina: imagens e mitos do fascínio tecnológico. Eikon: Journal on Semiotics and Culture, Covilhã, v. 1, n. 6, p.7-16, dez. 2019a. Disponível em: http://ojs.labcom-ifp.ubi.pt/index.php/eikon/article/ view/710. Acesso em: 31 jan. 2020. 
. Não são os professores que estão doutrinando jovens. São os gurus de redes sociais. Observatório da Imprensa. São Paulo, n. 1032. 5 fev. 2019b. Disponível em: http://observatoriodaimprensa.com.br/dilemas-contemporaneos/nao-sao-os-professores-que-estao-doutrinando-jovens-sao-os-gurus-de-redes-sociais. Acesso em 31 jan. 2020.

- Youtubers já são mais influentes do que jornalistas. Observatório da Imprensa. São Paulo, n. 1033, 16 abr. 2019c. Disponível em: http://observatoriodaimprensa.com.br/internet/youtubers-ja-sao-mais-influentes-do-que-jornalistas. Acesso em: 31 jan. 2020.

- Comunicação das universidades ainda despreza interesse público. Observatório da Imprensa. São Paulo, n.1042, 18 jun. 2019d. Disponível em: http:// observatoriodaimprensa.com.br/ciencia/comunicacao-das-universidades-ainda-despreza-interesse-publico. Acesso em: 31 jan. 2020.

GUMS, Elyson; IOSCOTE, Fabia; SPENASSATTO, Gabriel; JOHN, Valquiria Michela. Pesquisa exploratória de podcasts brasileiros voltados à Divulgação Científica. In: CONGRESSO DE CIÊNCIAS DA COMUNICAÇÃO NA REGIÃO SUL, 20. 2019, Porto Alegre. Anais... Porto Alegre: Intercom, 2019. p.1-14. Disponível em: https://portalintercom.org.br/anais/sul2019/ resumos/R65-1708-1.pdf. Acesso em: 04 set. 2020.

LIMA, Telma Cristiane Sasso de; MIOTO, Regina Célia Tamaso. Procedimentos metodológicos na construção do conhecimento científico: a pesquisa bibliográfica. Revista Katálysis, v. 10, n. SPE, p. 37-45, 2007.

LUIZ, Lúcio; ASSIS, Pablo de. O Podcast no Brasil e no Mundo: um caminho para a distribuição de mídias digitais. In: CONGRESSO BRASILEIRO DE CIÊNCIAS DA COMUNICAÇÃO, 33. 2010, Caxias do Sul. Anais... São Paulo: Intercom, 2010. p.1-15. Disponível em: http://www. intercom.org.br/papers/nacionais/2010/resumos/R5-0302-1.pdf. Acesso em: 04 set. 2020.

MASSARANI, Luisa; MOREIRA, Ildeu de Castro. Science communication in Brazil: A historical review and considerations about the current situation. Anais da Academia Brasileira de Ciências, v. 88, n. 3, p. 1577-1595, 2016.

MENDES, Lênio Bronzeado. Contribuições da linguagem radiofônica em podcast de divulgação científica: o caso do programa "Oxigênio". 2019. 1 recurso online (149p.). Dissertação (mestrado) - Universidade Estadual de Campinas, Instituto de Estudos da Linguagem, Campinas, SP.

MORAIS, J. I. de; ABONIZIO, H. Q.; TAVARES, G. M.; FONSECA, A. A. da; BARBON JUNIOR, S. Deciding among fake, satirical, objective and legitimate news: a multi-label classification system. In: BRAZILIAN SYMPOSIUM ON INFORMATION SYSTEMS, 15, 2019, Aracaju. Proceedings of the XV Brazilian Symposium on Information Systems. Porto Alegre: Sbc, 2019. p. 1-8.

MOREIRA, I. C.; MASSARANI, L. Aspectos históricos da Divulgação Científica no Brasil. In: MASSARANI, L.; MOREIRA, I. C.; BRITO, F. Ciência e Público. Caminhos da Divulgação Científica no Brasil. Rio de Janeiro, Casa da Ciência/UFRJ: Editora UFRJ, 2002. p. 43-64.

RAMOS, Vanessa Likoski. YouTube e a disseminação de conteúdo científico na Internet: perspectivas sobre critérios de qualidade em vlogs. Porto Alegre, 2017. 
REALE, Manuella Vieira. O sabor do saber: divulgação científica em interação no YouTube. 2018. 165 f. Dissertação (Mestrado em Comunicação e Semiótica) - Programa de Estudos Pós-Graduados em Comunicação e Semiótica, Pontifícia Universidade Católica de São Paulo, São Paulo, 2018.

REALE, Manuella Vieira; MARTYNIUK, Valdenise Leziér. Divulgação Científica no Youtube: a construção de sentido de pesquisadores nerds comunicando ciência. In: CONGRESSO BRASILEIRO DE CIÊNCIAS DA COMUNICAÇÃO, 39. 2016, São Paulo. Anais... São Paulo: Intercom, 2016. p. 1-15. Disponível em: https://portalintercom.org.br/anais/nacional2016/resumos/R11-0897-1.pdf. Acesso em: 04 set. 2020.

RIEDER, Bernhard. YouTube Data Tools (Version 1.11) [Software]. Disponível em: https:// tools.digitalmethods.net/netvizz/youtube/. Acesso em: 18 ago. 2020.

SILVA, A. C. P.; NASCIMENTO, F. C.; FONSECA, A. A. da; RODRIGUES, I. A História no Youtube: democratização, vulgarização e falsos problemas para o conhecimento histórico. Outras Fronteiras: revista discente do programa de pós-graduação em história da UFMT. Cuiabá, v.5, n. 2, p.143-158, 2019. Disponível em: http://ppghis.com/outrasfronteiras/index. php/outrasfronteiras/article/view/339. Acesso em 31 jan. 2020.

VELHO, Raphaela Martins Guedes de Azevedo. O papel dos vídeos de ciência na divulgação científica: o caso do projeto ScienceVlogs Brasil. 2019. 1 recurso online (174 p.). Dissertação (mestrado) - Universidade Estadual de Campinas, Instituto de Estudos da Linguagem, Campinas, SP.

VOGT, Carlos. A espiral da cultura científica. Revista ComCiência, v. 45, 2003. Disponível em: http://www.comciencia.br/dossies-1-72/reportagens/cultura/cultura01.shtml. Acesso em: 23 mar. 2020. 


\section{André Azevedo da Fonseca}

Professor adjunto no Centro de Educação, Comunicação e Artes (CECA) da Universidade Estadual de Londrina (UEL). Doutor em História (Unesp) com pós-doutorado no Programa Avançado de Cultura Contemporânea (UFRJ).

E-mail: andre.azevedo@uel.br

\section{Leonardo Mendes Bueno}

Estudante de Jornalismo na Universidade Estadual de Londrina. Bolsista de Iniciação Científica (CNPq).

E-mail:leo_leomb@hotmail.com 